\title{
Frecuencia de depresión y ansiedad no diagnosticadas en pacientes con diabetes mellitus tipo 2 que acuden a la consulta externa de un hospital general de Lima, Perú.
}

Frequency of undiagnosed depression and anxiety in patients with Type 2 Diabetes Mellitus attended at the outpatient clinic of a General Hospital in Lima, Peru.

Johan Rosas-Matías ${ }^{1, a}$, Alexander Villanueva-Bejarano ${ }^{1, a}$, Francesco Pantani-Romero ${ }^{1, a}$, Antonio Lozano$\operatorname{Vargas}^{2,3,4, b}$, Ray Ticse-Aguirre ${ }^{5,6,6, d}$.

\section{RESUMEN}

Antecedentes: Las poblaciones de pacientes con diabetes mellitus tipo 2 (DM2) generalmente tienen mayor frecuencia de enfermedades psiquiátricas que la población general. Objetivo: Identificar la frecuencia de pacientes con depresión y ansiedad no diagnosticadas en pacientes con DM2 que acuden a la consulta externa de un hospital general de Lima. Material y métodos: Se realizó un estudio descriptivo transversal en pacientes con diagnóstico de DM2, a fin de determinar la presencia de depresión y ansiedad de acuerdo a la Escala de Psicopatología Depresiva (EPD-6) y la Escala de Ansiedad de Lima (EAL-20). Resultados: En la muestra de 327 pacientes, el 23,9\% presentaron depresión, el 32,7\% ansiedad y el 7,95\% suicidalidad, en tanto que un 5,5 presentaron síntomas de depresión y ansiedad. El 17,94\% de pacientes con depresión y el 16,82\% de pacientes con ansiedad presentaron suicidalidad. Las mujeres tuvieron una frecuencia de ansiedad de $39,9 \%$ y los varones, de $16,7 \%$ ( $<<0,01)$. La mediana de edad de los pacientes con depresión fue de 59 años (IQT: 51-64) versus 62 años (IQT: 54-69) de los pacientes sin depresión $(\mathrm{p}=0,038)$. Conclusiones: Casi una cuarta parte $(23,9 \%)$ de los pacientes con DM2 mostraron evidencia de depresión, y un tercio $(33,7 \%)$ presentaron ansiedad. Además, el sexo femenino y la edad parecen estar significativamente relacionados a ansiedad y depresión, respectivamente.

PALABRAS CLAVE: Depresión, ansiedad, diabetes mellitus.

\footnotetext{
Universidad Peruana Cayetano Heredia, Facultad de Medicina Alberto Hurtado. Lima, Perú.

2 Universidad Peruana Cayetano Heredia, Facultad de Medicina Alberto Hurtado, Sección de Psiquiatría y Salud Mental. Lima, Perú.

3 Hospital Cayetano Heredia, Servicio de Neuro-Psiquiatría. Lima, Perú.

4 Clínica Anglo Americana. Lima, Perú.

5 Universidad Peruana Cayetano Heredia, Facultad de Medicina Alberto Hurtado, Departamento de Clínicas Médicas. Lima, Perú.

6 Hospital Cayetano Heredia, Servicio de Endocrinología. Lima, Perú.

a $\quad$ Médico general; ${ }^{\mathrm{b}}$ Médico Psiquiatra; ${ }^{\mathrm{c}}$ Médico Internista y Endocrinólogo; ${ }^{\mathrm{d}}$ Maestría en epidemiología clínica.
} 
Frecuencia de depresión y ansiedad no diagnosticadas en pacientes con diabetes mellitus tipo 2.

\section{SUMMARY}

Background: The populations of patients with Type 2 Diabetes Mellitus (DM2) generally have a higher frequency of psychiatric illnesses than the general population. Objective: To identify the frequency of undiagnosed depression and anxiety in patients with DM2 who attended the outpatient clinic of a general hospital in Lima. Material and methods: A descriptive, cross-sectional study was conducted on patients with DM2, among whom the presence of depression and anxiety was determined by using the Depressive Psychopathology Scale (EPD-6) and the Lima Anxiety Scale (LAS-20). Results: From 327 patients included in the study, 23.9\% had depression, 32.7\% anxiety and $7.95 \%$ suicidality, whereas $5.5 \%$ presented symptoms of both, depression and anxiety. Additionally, $17.94 \%$ of patients with depression, and $16.82 \%$ of those with anxiety presented evidence of suicidality. $39.9 \%$ of women had anxiety compared to $16.7 \%$ of men $(\mathrm{p}<0.01)$. The median age of patients with depression was 59 years (IQT: 51-64) versus 62 years (IQT: 54-69) among patients without depression ( $\mathrm{p}=0.038)$. Conclusions: Almost a quarter $(23.9 \%)$ of the patients with DM2 had depression, and a third (33.7\%) presented anxiety. In addition, female sex and age appear to be significantly related to anxiety and depression, respectively.

KEYWORDS: Depression, anxiety, diabetes mellitus.

\section{INTRODUCCIÓN}

La diabetes mellitus está catalogada como una de las enfermedades no transmisibles más frecuentes a nivel mundial con un aumento en su prevalencia de $4,7 \%$ a $8,5 \%$ en los últimos 40 años, siendo la diabetes mellitus tipo 2 (DM2) el tipo más frecuente (1). En el Perú, Seclén y colaboradores reportaron una prevalencia de DM2 de 7\% en pacientes mayores de 25 años (2); a pesar de ello, hasta el día de hoy no existen políticas en salud en nuestro país que intenten controlar el avance de la enfermedad o reducir sus factores de riesgo (3).

Por otro lado, la depresión afecta a más de 300 millones de personas en el mundo y es la principal causa mundial de discapacidad. En casos de mayor severidad puede llevar al suicidio (1). En el Perú, según la Encuesta Demográfica y de Salud Familiar (ENDES), entre los años 2014 y 2015, la prevalencia estimada de depresión fue de $14 \%$, utilizando como instrumento de tamizaje el Patient Health Questionary-9 (PHQ-9) (4).

Así mismo, se ha estimado que aproximadamente el $10 \%$ de la población mundial padece de algún trastorno de ansiedad, de los cuales solo el 27,6\% recibieron tratamiento (5). En el Perú, este trastorno psiquiátrico tiene una prevalencia actual de 1,9\%, sin embargo, no es diagnosticado oportunamente y el tratamiento generalmente está postergado (2).

Los pacientes con DM2 pueden presentar trastornos del ánimo y de ansiedad. Estos trastornos psiquiátricos conllevan a un estrés psicológico crónico, pueden presentar hipercortisolismo por activación del eje hipotálamo - pituitario - suprarrenal; y por otro lado, el sistema nervioso simpático aumenta la agregación plaquetaria y respuesta inflamatoria, teniendo efectos a nivel del páncreas disminuyendo la sensibilidad de la insulina y exacerbando intolerancia a la glucosa (6).

Existen diversos estudios que muestran una frecuencia muy variable de estos trastornos psiquiátricos en la población con DM2, encontrando prevalencias de $30 \%$ - $65 \%$ para depresión $(6,7,8)$ y de $14 \%-80 \%$ en lo correspondiente a ansiedad (9). Generalmente, la frecuencia de trastornos psiquiátricos en la población con DM2 es mayor que en la población general. Sin embargo, los valores de las frecuencias pueden ser muy variables según la población estudiada, criterios de diagnóstico, herramienta utilizada para la medición y clasificación, etc. Por ejemplo, en un estudio realizado en Lima Metropolitana en el 2015, la prevalencia de depresión en pacientes con DM2 fue de $5,77 \%(10)$.

En una revisión sistemática de tipo meta-análisis se incluyeron 28 estudios analíticos observacionales de cohortes y se concluyó que el riesgo de suicidio se incrementa significativamente en pacientes con DM2 en general (11).

Para el diagnóstico de trastornos psiquiátricos, uno de los estándar de oro es la Structured Clinical Interview for DSM Disorders (SCID) y diferentes instrumentos auto-aplicables que han elaborado han demostrado ser de utilidad al contrastarse con dicho estándar de oro (12). Para el tamizaje de depresión, fueron 
desarrollados el inventario de depresión de Beck, la escala Center for Epidemiological Studies-Depression (CES-D), la Escala Auto-aplicable de Depresión de Zung (ZSDS) y la Escala de Psicopatología Depresiva (EPD) (13).

En un estudio en el Perú se elaboró versiones cortas de la EPD buscando disminuir el tiempo de aplicación de esta escala sin perder su eficacia en la detección de la presencia de depresión, obteniéndose la EPD-6, la cual demostró cumplir con estos requisitos (14).

De la misma manera, también en el Perú, se desarrolló la Escala de Ansiedad de Lima de 130 ítems (EAL-130) (15). Y en un estudio posterior, se validó una versión más corta seleccionando 20 ítems (EAL$20)$, los cuales lograron predecir adecuadamente los resultados obtenidos por la escala inicial (16).

La suicidalidad, según la Columbia Classification Algorithm of Suicide Assessment (C-CASA) incluye términos que hacen referencia a la conducta suicida (pensamiento, ideación, intento suicida) e ideas pasivas de muerte (17). Si bien aún es controversial su uso en estudios psiquiátricos, nos brinda información acerca de los factores de riesgo en las personas para presentar conducta suicida e ideas pasivas de muerte.

La importancia de realizar este estudio radica en la necesidad de mostrar a los profesionales de salud, específicamente a los que están involucrados en el tratamiento de los pacientes con DM2, la frecuencia de la depresión y ansiedad no diagnosticada en esta población. La depresión y ansiedad al no ser diagnosticadas y tratadas adecuadamente influyen negativamente en el autocuidado de la salud reflejándose en controles glucémicos inadecuados, disminuyendo la calidad de vida y funcionalidad (10).

El presente estudio tuvo como objetivo determinar la frecuencia de depresión y ansiedad no diagnosticadas en pacientes con DM2 que acuden a la consulta externa de un hospital general de Lima; y a su vez determinar la presencia de suicidalidad y describir las características de la población estudiada.

\section{MATERIAL Y MÉTODOS}

El diseño del presente estudio es transversal y descriptivo, en una población de pacientes con diagnóstico de DM2 que acuden al consultorio externo de endocrinología del Hospital Cayetano Heredia $(\mathrm{HCH})$.
Se consideraron los siguientes criterios de inclusión: a) pacientes con diagnóstico de DM2; b) con primaria completa; y c) que hayan aceptado participar del estudio mediante la firma del consentimiento informado.

Se consideraron los siguientes criterios de exclusión: a) pacientes con alguna complicación crónica grave diagnosticada tales como cardiopatías, amputaciones, enfermedad renal crónica y condiciones similares; y b) diagnóstico reciente y/o tratamiento actual de depresión y/o ansiedad consignado en la historia clínica.

El tipo de muestreo fue no probabilístico por conveniencia. Se acudió al consultorio externo del servicio de endocrinología, de lunes a sábados en dos turnos (mañana y tarde), durante el mes de febrero del 2019. Previa firma de consentimiento informado, fueron entrevistados 337 pacientes con diagnósticos de DM2 verificados en sus historias clínicas. Del total de encuestas completadas, 10 presentaron incongruencias en el llenado tales como datos faltantes, marcar 2 alternativas a la vez, y similares; por lo que fueron descartadas, con lo que se evaluaron 327, siendo esta la muestra total.

Las variables principales del estudio fueron:

Depresión: Puntaje mayor o igual a 8 al aplicar la EPD - 6.

Ansiedad: Puntaje mayor o igual a 10 al aplicar la $\mathrm{EAL}-20$.

Suicidalidad: Corresponde a una alternativa diferente a la "a" del ítem 20 de la EPD - 20.

Se aplicó las siguientes escalas de medición:

a) Escala breve para la evaluación de sintomas depresivos: $E P D-6(14)$

Escala auto - aplicable que consta de 6 ítems de la EPD - 20. La puntuación es numérica, siendo: "a" $=0$; "b" =1; "c" =2; "d" =3; a excepción de la pregunta 3 en la cual "a" $=3$; "b" $=2$; "c" $=1$; "d" $=0$. El punto de corte para predecir presencia de sintomatología depresiva es de 8 . Este punto de corte fue establecido por los autores teniendo en cuenta que presentaba el mayor índice de Youden $(0,5387)$ consiguiendo una sensibilidad de $80 \%$ y especificidad de $74 \%$. 
Frecuencia de depresión y ansiedad no diagnosticadas en pacientes con diabetes mellitus tipo 2.

Tabla 1. Características sociodemográficas de la muestra estudiada. Frecuencia de depresión y ansiedad no diagnosticados en pacientes con diabetes mellitus tipo 2 que acuden a la consulta externa de Endocrinología, HCH. 2019.

\begin{tabular}{|c|c|c|c|}
\hline Características & & $\mathrm{n}$ & $\%$ \\
\hline \multirow{2}{*}{ Sexo } & Femenino & 231 & 70,64 \\
\hline & Masculino & 96 & 29,36 \\
\hline \multirow{6}{*}{ Edad } & $21-31$ años & 6 & 1,83 \\
\hline & $32-42$ años & 15 & 4,59 \\
\hline & $43-53$ años & 64 & 19,57 \\
\hline & $54-64$ años & 120 & 36,70 \\
\hline & $65-75$ años & 100 & 30,58 \\
\hline & 76 años a más & 22 & 6,73 \\
\hline \multirow{5}{*}{ Estado Civil } & Soltero & 84 & 25,69 \\
\hline & Conviviente & 53 & 16,21 \\
\hline & Casado & 125 & 38,23 \\
\hline & Divorciado/Separado & 22 & 6,73 \\
\hline & Viudo & 43 & 13,15 \\
\hline \multirow{5}{*}{ Nivel Académico } & Primaria Completa & 120 & 36,70 \\
\hline & Secundaria Incompleta & 47 & 14,37 \\
\hline & Secundaria Completa & 106 & 32,30 \\
\hline & Superior Incompleta & 15 & 4,59 \\
\hline & Superior Completa & 39 & 11,93 \\
\hline \multirow{4}{*}{ Ocupación } & Ama de Casa & 179 & 54,74 \\
\hline & Trabajador Dependiente & 23 & 7,03 \\
\hline & Trabajador Independiente & 93 & 28,44 \\
\hline & Desocupado & 32 & 9,79 \\
\hline \multirow{4}{*}{ Tiempo de diagnóstico } & Menos de 10 años & 220 & 67,28 \\
\hline & $11-20$ años & 72 & 22,02 \\
\hline & $21-30$ años & 28 & 8,56 \\
\hline & $31-40$ años & 7 & 2,14 \\
\hline
\end{tabular}

b) Escala breve para la evaluación de síntomas ansiosos: $E A L-20$ (16)

Escala auto - aplicable que consta de 20 ítems de la EAL-130. La puntuación es numérica, siendo "Sí" = 1; "No" = 0; a excepción de la pregunta 18 en la cual "Sí" = $0 ;$ "No" = 1. El punto de corte para predecir presencia de sintomatología ansiosa es de 10. Este punto de corte fue establecido por los autores teniendo en cuenta que presentaba el mayor índice de Youden $(0,369)$ consiguiendo una sensibilidad de $69 \%$ y especificidad de $68 \%$ para la identificación de ansiedad moderada o mayor.

c) Ítem 20 de la EPD-20 sobre suicidalidad (18)

Es la pregunta 20 de la EPD-20, que explora la presencia de suicidalidad. La calificación es dicotómica, siendo "a" = No presencia de suicidalidad; "b", "c", "d", "e" = Presencia de suicidalidad. 
Los datos finales fueron recolectados y analizados mediante los programas Microsoft Excel 2016 ® y STATA v. 14 ®.

Las variables cualitativas fueron presentadas como frecuencias, las variables cuantitativas como medias o medianas según su distribución sea normal o no, con sus respectivas desviaciones estándar o intervalos intercuartiles 25 y 75, respectivamente. Para buscar relación entre sexo, ocupación, nivel académico y estado civil; con depresión, ansiedad y suicidalidad, se utilizó la prueba de chi cuadrado. Mientras que para el análisis estadístico entre edad y tiempo de diagnóstico; con depresión, ansiedad y suicidalidad, se utilizó la prueba de U de Mann-Whitney. La distribución de las variables cuantitativas no fue normal, lo cual fue demostrado mediante la prueba de Shapiro-Wilk.
El presente estudio fue aprobado por el Comité Institucional de Ética en Investigación de la Universidad Peruana Cayetano Heredia (UPCH).

Cabe resaltar que los pacientes con presencia positiva de la variable de suicidalidad en la escala fueron derivados y acompañados por uno de los investigadores al consultorio de psiquiatría para asegurar su atención especializada en salud mental.

\section{RESULTADOS}

Las características socio-demográficas de la población estudiada se pueden apreciar en la tabla 1. De la muestra estudiada se encontró que el 23,85\% presentaron depresión, el 32,72\% ansiedad y el 7,95\% suicidalidad (tabla 2); así mismo, el 5,5\% presentaron síntomas de depresión y de ansiedad (tabla 3).

Tabla 2. Frecuencias de depresión, ansiedad y suicidalidad no diagnosticados en pacientes con diabetes mellitus tipo 2 que acuden a la consulta externa de Endocrinología, HCH. 2019.

\begin{tabular}{llcc}
\hline & & $\mathbf{n}$ & $\mathbf{\%}$ \\
\hline \multirow{2}{*}{ Depresión } & No & 249 & 76,15 \\
& Sí & 78 & 23,85 \\
\multirow{2}{*}{ Ansiedad } & No & 220 & 67,28 \\
\multirow{2}{*}{ Suicidalidad } & Sí & 107 & 32,72 \\
& No & 301 & 92,05 \\
& Sí & 26 & 7,95 \\
\hline
\end{tabular}

Tabla 3. Frecuencias de ansiedad según la presencia o no de depresión. Consultorio de Endocrinología, HCH. 2019.

\begin{tabular}{llcc}
\hline & & Sin Depresión & Con Depresión \\
\hline Ansiedad & No & $212(64,8 \%)$ & $8(2,4 \%)$ \\
$(\mathrm{p}<0,001)$ & Sí & $89(27,2 \%)$ & $18(5,5 \%)$ \\
\hline
\end{tabular}

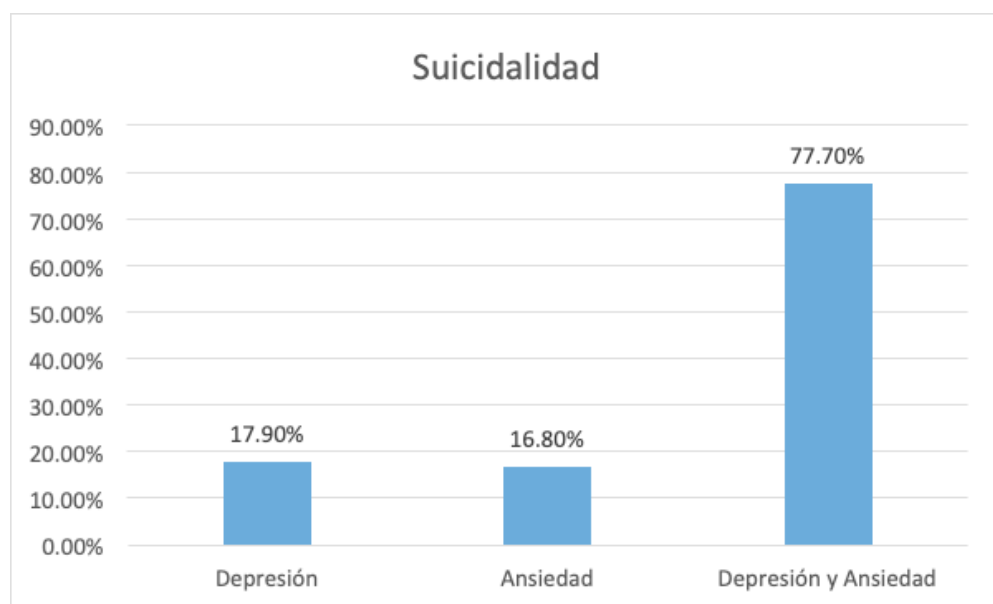

Gráfico 1. Frecuencias de suicidalidad según depresión y ansiedad. Consultorio de Endocrinología, HCH. 2019. 
Frecuencia de depresión y ansiedad no diagnosticadas en pacientes con diabetes mellitus tipo 2.

Tabla 4. Frecuencias de ansiedad y depresión según sexo. Frecuencia de depresión y ansiedad no diagnosticados en pacientes con diabetes mellitus tipo 2 que acuden a la consulta externa de Endocrinología, HCH. 2019.

\begin{tabular}{llll}
\hline & & $\begin{array}{l}\text { Con ansiedad } \\
(\mathbf{p}<\mathbf{0 , 0 0 1})\end{array}$ & $\begin{array}{l}\text { Con depresión } \\
(\mathbf{p}=\mathbf{0 , 2 6 7 )}\end{array}$ \\
\hline \multirow{3}{*}{ Sexo } & $\begin{array}{l}\text { Masculino } \\
\mathrm{N}=96(100 \%)\end{array}$ & $16(16,7 \%)$ & $19(19,8 \%)$ \\
& $\begin{array}{l}\text { Femenino } \\
\mathrm{N}=231(100 \%)\end{array}$ & $91(39,9 \%)$ & $59(25,6 \%)$ \\
\hline
\end{tabular}

Tabla 5. Frecuencias de ansiedad y depresión según ocupación. Consultorio de Endocrinología, HCH. 2019.

\begin{tabular}{llcc}
\hline & \multicolumn{1}{c}{$\begin{array}{l}\text { Con ansiedad } \\
(\mathbf{p}<\mathbf{0 , 0 0 1})\end{array}$} & $\begin{array}{l}\text { Con depresión } \\
(\mathbf{p}=\mathbf{0 , 7 3 0 )}\end{array}$ \\
\hline \multirow{2}{*}{ Ocupación de casa } & $74(41,4 \%)$ & $47(26,2 \%)$ \\
& $\begin{array}{l}\mathrm{N}=179(100 \%) \\
\text { Trabajador dependiente }\end{array}$ & $10(43,5 \%)$ & $5(21,7 \%)$ \\
& $\begin{array}{l}\text { Trabajador independiente } \\
\mathrm{N}=93(100 \%)\end{array}$ & $19(20,5 \%)$ & $19(20,5 \%)$ \\
$\begin{array}{l}\text { Desocupado } \\
\mathrm{N}=32(100 \%)\end{array}$ & $4(12,5 \%)$ & $7(21,8 \%)$ \\
\hline
\end{tabular}

Tabla 6. Frecuencias de depresión, ansiedad y suicidalidad según la mediana del tiempo de diagnóstico. Consultorio de Endocrinología, Hospital Cayetano Heredia. 2019.

\begin{tabular}{ccccc}
\hline & Depresión & Ansiedad & Suicidalidad \\
\hline & $\begin{array}{c}\text { Menos de 10 años } \\
(\mathrm{N} 1)\end{array}$ & $51(23,1 \%)^{*}$ & $67(30,4 \%)^{*}$ & $16(7,2 \%)^{*}$ \\
$\begin{array}{c}11-20 \text { años } \\
\text { Tiempo de }\end{array}$ & $21(29,1 \%)^{*}$ & $27(37,5 \%)^{*}$ & $8(11,1 \%)^{*}$ \\
Diagnóstico & $\begin{array}{c}21-30 \text { años } \\
(\mathrm{N} 3)\end{array}$ & $6(21,4 \%)^{*}$ & $8(28,5 \%)^{*}$ & $1(3,5 \%)^{*}$ \\
& $\begin{array}{c}31 \text { años a más } \\
(\mathrm{N} 4)\end{array}$ & $0(0 \%)^{*}$ & $5(71,4 \%)^{*}$ & $1(14,2 \%)^{*}$ \\
\hline
\end{tabular}

* Los porcentajes entre paréntesis corresponden a la frecuencia relativa de cada trastorno con respecto al total de pacientes ubicados en dicho grupo (N1, N2, N3 y N4).

En el gráfico 1 se observa a los pacientes con depresión $(\mathrm{N}=78)$ y con ansiedad $(\mathrm{N}=107)$ según si presentaron o no suicidalidad. Se puede observar que el $17,9 \%$ de pacientes con depresión y el $16,8 \%$ de pacientes con ansiedad presentaron suicidalidad. La depresión y/o la ansiedad se encuentran relacionadas a la suicidalidad $(\mathrm{p}<0,001)$.

En relación al sexo, se encontró que los pacientes de sexo femenino presentaron mayor frecuencia de ansiedad, llegando al 39,9\% versus 16,7\% $(\mathrm{p}<0,01)$. No hubo diferencias con respecto a la depresión (tabla 4).
En cuanto a la ocupación, se determinó que los trabajadores dependientes presentaron más ansiedad, la que correspondía al 43,47\% $(\mathrm{p}<0,01)$ (tabla 5).

La mediana de edad de los pacientes con depresión fue de 59 años (IQT:51-64) versus 62 años (IQT:54-69) de los pacientes sin depresión $(\mathrm{p}=0,038)$. La mediana de la edad de los pacientes con ansiedad fue de 60 años (26-83 años). No hubo diferencias estadísticamente significativas con los pacientes sin ansiedad.

Se encontró que el grupo con tiempo de diagnóstico de 11 a 20 años fue el que presentó mayor frecuencia 
de depresión; sin embargo, el grupo de 31 años a más presentaron mayor frecuencia de ansiedad y suicidalidad (tabla 6).

No se encontró relación entre las variables nivel académico, estado civil y tiempo de diagnóstico y las variables principales $(\mathrm{p}>0,05)$.

\section{DISCUSIÓN}

Existen estudios donde se ha descrito que los pacientes con DM2, comparados con la población general, tienen dos veces más riesgo de desarrollar trastornos del ánimo y de ansiedad (19). Bajo esa premisa, se han realizado muchos estudios clínicos y epidemiológicos donde se ha observado una clara asociación entre DM2, ansiedad y depresión, siendo estos de gran importancia en la toma de decisiones terapéuticas por sus efectos negativos en el control metabólico y adherencia al tratamiento (19).

La alta frecuencia $(23,85 \%)$ de depresión en pacientes con DM2 en el presente trabajo concuerda con la prevalencia a nivel nacional que es del $20 \%$, reportada por el Instituto Nacional de Salud (20); sin embargo, es menor que la observada en otros estudios nacionales de depresión y DM2, quienes señalan frecuencias de $31 \%$ - 45\% $(21,22)$, y que en estudios internacionales quienes encontraron frecuencias que varían entre $24 \%$ - $82 \%(23,24)$. Esta diferencia puede deberse a que en dichos estudios se emplearon otras escalas de medición, tales como la escala de Zung, el Inventario de Depresión de Beck y la MINI.

Otros estudios, señalan que el impacto de estos trastornos psiquiátricos se puede ver reflejado en la respuesta al tratamiento a través de los valores de hemoglobina glicosilada, glicemia, medidas antropométricas entre otros $(19,21,23)$, las cuales no fueron utilizadas en el presente estudio debido al registro incompleto de resultados de laboratorio en las historias clínicas.

En lo que respecta a ansiedad, el 32,72\% obtenido se encuentra dentro del rango descrito en la bibliografía nacional e internacional revisada, en las que se evidencian frecuencias muy variables que van desde $14 \%$ hasta $80 \%(21,25-27)$.

La frecuencia de pacientes con DM2 con suicidalidad fue $7,9 \%$. No se encontró estudios previos que analicen la relación entre estas, por lo que su hallazgo representa un dato importante que debe tomarse a consideración por parte de los médicos tratantes del servicio de Endocrinología, ya que el diagnostico de DM2 tiene un impacto emocional significativo en el individuo, produciendo muchas veces pensamientos negativos y hasta el suicidio $(11,17,28)$.

Se ha demostrado que aquellas personas con DM2 tienen más probabilidad de desarrollar trastornos psiquiátricos debido a diversos factores, entre ellos el estrés, cronicidad de la enfermedad, el autocuidado y el tratamiento a seguir (6). Además, en el presente estudio se encontró que los factores relacionados al desarrollo de depresión y ansiedad son el sexo, la ocupación y edad.

En este estudio el sexo femenino presenta más frecuencia de ansiedad. Esto puede deberse a las diferencias en el estilo de vida, ya que las mujeres pasan mayor tiempo en el hogar, lo que conlleva a una menor actividad física y malos hábitos alimenticios (29). Las mujeres presentan también un factor hormonal, puesto que después de los 50 años, el estrógeno disminuye gradualmente en las mujeres, lo cual conlleva a cambios en su bienestar general (29).

En cuanto a ocupación, la mayor frecuencia de ansiedad se dio en los trabajadores dependientes, hallándose que estas variables están relacionadas. Las investigaciones indican que la pobreza, la dificultad financiera y otros problemas económicos, pueden aumentar el riesgo de desarrollar ansiedad (30).

En cuanto a la edad, se encontró que está relacionada con la presencia de depresión, hallándose que los más jóvenes presentan mayor frecuencia de este trastorno; sin embargo, estos resultados no coinciden con otros estudios donde señalan que la depresión es más prevalente con el aumento de la edad $(31,32)$. Probablemente se deba a que el impacto emocional de padecer diabetes mellitus tipo 2 en pacientes jóvenes tenga mayor repercusión que en los pacientes adultos (6).

En vista de los resultados obtenidos, es preocupante la alta frecuencia del sub-diagnóstico de los trastornos psiquiátricos en los pacientes con DM2, por ello, se recomienda el uso de escalas de tamizaje como las empleadas en el presente estudio (EPD-6, EAL-20) para mejorar los objetivos de tratamiento en estos pacientes y a su vez en su calidad de vida. 
Frecuencia de depresión y ansiedad no diagnosticadas en pacientes con diabetes mellitus tipo 2.

Dentro de las limitaciones de este estudio se pueden tener en cuenta la omisión del control de la DM2 por medio de la hemoglobina glicosilada, mediciones antropométricas, el mal llenado de las historias clínicas y el pobre conocimiento de los mismos pacientes acerca de su condición de salud. Otra limitación a tomar en cuenta es el momento de aplicación de las encuestas, el cual generaba un grado de estrés en el paciente por encontrarse a la espera de ingresar al consultorio para ser atendido.

En conclusión, la cuarta parte de los pacientes $(23,9 \%)$ de los pacientes con DM2 tuvieron depresión, y un tercio $(33,7 \%)$ presentaron ansiedad. Además, el sexo femenino y la edad están relacionados a la ansiedad y depresión respectivamente. Se recomienda que debido a la elevada frecuencia de pacientes con DM2 que no tienen diagnóstico de depresión ni de ansiedad, se debe utilizar escalas de tamizaje breves, como las utilizadas en este estudio, y así poder derivar al paciente al médico especialista como parte de la atención integral al paciente.

Conflictos de interés: Los autores declaran no tenerlos. Declaración de financiamiento: Financiado por los autores.

\section{Correspondencia:}

Ray Ticse Aguirre

Servicio de Endocrinología, Hospital Cayetano Heredia.

Av. Honorio Delgado 262, San Martin de Porres, Lima, Perú.

Correo electrónico: ray.ticse@upch.pe

\section{REFERENCIAS BIBLIOGRÁFICAS}

1. Organización mundial de la salud. Depresión. Ginebra: Organización Mundial de la Salud; 2018. (Fecha de acceso 11 de enero del 2019) Disponible en: https://www.who.int/es/news-room/fact-sheets/ detail/depression

2. Seclén SN, Rosas ME, Arias AJ, Huayta E, Medina CA. Prevalence of diabetes and impaired fasting glucose in Peru: report from PERUDIAB, a national urban population-based longitudinal study. BMJ Open Diabetes Research \& Care. 2015;3(1): e000110.

3. Organización Mundial de la Salud. Perfiles de los países para la diabetes. Ginebra: Organización mundial de la salud; 2016. (Fecha de acceso 11 de enero del 2019) Disponible en: https://www.who.int/ diabetes/country-profiles/per_es.pdf?ua $=1$

4. Martina M, Ara MA, Gutiérrez C, Nolberto V, Piscoya
J. Depresión y factores asociados en la población peruana adulta mayor según la ENDES 2014-2015. An Fac Med. 2017;78(4):393-7.

5. Alonso J, Liu Z, Evans-Lacko S, et al. Treatment gap for anxiety disorders is global: Results of the World Mental Health Surveys in 21 countries. Depress Anxiety. 2018; 35(3):195-208.

6. Antúnez M, Bettio A. Depresión en pacientes con diabetes tipo 2 que acuden a una consulta externa de medicina interna. Acta Med Colomb. 2016; 41:102110.

7. Van Dijk SEM, Adriaanse MC, van der Zwaan L, Bosmans JE, van Marwijk HWJ, van Tulder MW, et al. Measurement properties of depression questionnaires in patients with diabetes: a systematic review. Qual Life Res. 2018; 27(6):1415-30.

8. Udovichenko OV, Maximova NV, Amosova MV, Yunilaynen OA, Berseneva EA, Starostina EG. Prevalence and prognostic value of depression and anxiety in patients with diabetic foot ulcers and possibilities of their treatment. Curr Diabetes Rev. 2017; 13(1):97-106.

9. Rivas-Acuña V, Gracias-Barjau H, Cruz Leon-A, et al. Prevalencia de ansiedad y depresión en las personas con diabetes mellitus tipo 2. Salud en Tabasco. 2011; 17(1):30-35.

10. Arias KB. Prevalencia de depresión y sus factores asociados en pacientes con diabetes mellitus que acuden a consulta externa de hospitales generales y centros de salud de Lima Metropolitana y el Callao -2015.Tesis de Bachiller en Medicina. Lima, Perú: Universidad Peruana Cayetano Heredia; 2018.

11. Wang B, An X, Shi X, Zhang JA. Management of endocrine disease: Suicide risk in patients with diabetes: a systematic review and meta-analysis. Eur J Endocrinol. 2017; 177(4): R169-R181. doi: 10.1530/ EJE-16-0952

12. Dunstan DA, Scott N, Todd AK. Screening for anxiety and depression: reassessing the utility of the Zung scales. BMC Psychiatry. 2017; 17(1): 329.

13. Ruiz-Grosso P, Loret de Mola C, Vega-Dienstmaier JM, Arévalo JM, Chavez K, Vilela A, et al. Validation of the Spanish Center for Epidemiological Studies Depression and Zung Self-Rating Depression Scales: a comparative validation study. PLoS ONE. 2012; 7(10): e45413.

14. Vega-Dienstmaier JM. Construcción de versiones cortas de la Escala de Psicopatología Depresiva para tamizaje de depresión mayor y sus características psicométricas. Rev Neuropsiquiatr. 2018; 81(3):15464.

15. Vega-Dienstmaier JM. Construcción de una nueva escala en español para medir ansiedad. Rev Neuropsiquiatr. 2011;74(2):230-241.

16. Lozano-Vargas A, Vega-Dienstmaier JM. Construcción y propiedades psicométricas de la 
Escala de Ansiedad de Lima de 20 ítems (EAL-20). Rev Neuropsiquiatr. 2018; 81(4):226-234.

17. Posner K, Oquendo MA, Gould M, Stanley B, Davies M. Columbia Classification Algorithm of Suicide Assessment (C-CASA): Classification of Suicidal Events in the FDA's Pediatric Suicidal Risk Analysis of Antidepressants. Am J Psychiatry. 2007; 164:1035-1043.

18. Vega-Dienstmaier JM, Stucchi-Portocarrero S, Valdez-Huarcaya N, Cabra-Bravo M, Zapata-Vega MI. The Depressive Psychopathology Scale: presentation and initial validation in a sample of Peruvian psychiatric patients. Rev Panam Salud Publica. 2011; 30: 317-326.

19. Fabián San Miguel MG, García Sancho F MC, Cobo Abreu C. Prevalencia de síntomas de ansiedad $\mathrm{y}$ depresión en pacientes con diabetes mellitus tipo $2 \mathrm{y}$ su asociación con el tipo de tratamiento, complicaciones de la diabetes y comorbilidades. Med Int Mex. 2010; 26(2):100-8.

20. Instituto Nacional de Salud. Unidad de Análisis y Generación de Evidencias en Salud Pública. Comorbilidad de la Depresión y Enfermedades Crónicas en la Población Peruana. Serie Análisis Secundario de Datos $\mathrm{N}^{\circ}$ 01-2016. Lima, Perú: Ministerio de Salud; 2016.

21. Constantino-Cerna A, Bocanegra-Malca M, LeónJiménez F, Díaz-Vélez C. Frecuencia de depresión y ansiedad en pacientes con diabetes tipo 2 atendidos en un hospital general de Chiclayo. Rev Med Hered. 2014; 24:196-203.

22. Huamán E. Depresión como factor asociado a control metabólico basado en hemoglobina glicosilada en pacientes diabéticos en el Hospital Regional Docente de Cajamarca noviembre 2016- enero 2017.Tesis de título profesional en Medicina. Cajamarca, Perú: Universidad Nacional de Cajamarca; 2017.

23. Instituto Nacional de Salud Mental. Estudio Epidemiológico de Salud Mental en Lima Metropolitana y Callao Replicación 2012: Informe General. Anales de Salud Mental. 2013;29: 1-392.
24. Wang L, Song R, Chen Z, Wang J, Ling F. Prevalence of depressive symptoms and factors associated with it in type 2 diabetic patients: a cross-sectional study in China. BMC Public Health. 2015;15:188.

25. Grigsby AB, Anderson RJ, Freedland KE, Clouse RE, Lustman PJ. Prevalence of anxiety in adults with diabetes: a systematic review. J Psychosom Res. 2002; 53: 1053-60.

26. Tovilla-Zárate C, Juárez-Rojop I, Peralta Jimenez Y. Prevalence of anxiety and depression among outpatients with type 2 diabetes in the Mexican population. PloS One. 2012; 7: e36887-e36887.

27. Mikaliūkštienė A, Žagminas K, Juozulynas A, et al. Prevalence and determinants of anxiety and depression symptoms in patients with type 2 diabetes in Lithuania. Med Sci Monit. 2014;20:182-90.

28. Ávila V, Apolinar G. Depresión y riesgo suicida en pacientes con diabetes mellitus tipo 2 en la unidad de salud del IMSS de Ciudad del Carmen, Campeche. Temas de Ciencia y Tecnología. 2016; 20(58): 37-44.

29. Sun N, Lou P, Shang Y. Prevalence and determinants of depressive and anxiety symptoms in adults with type 2 diabetes in China: a cross-sectional study. BMJ Open. 2016; 6(8):e012540.

30. Organización Mundial de la Salud. Suicidio. Ginebra: Organización Mundial de la Salud; 2019. (Fecha de acceso 11 de enero del 2019) Disponible en: https:// www.who.int/es/news-room/fact-sheets/detail/ suicide

31. Urrutia D, Segura R. Sintomatología depresiva y diabetes mellitus tipo 2 en una muestra ambulatoria de un hospital de las Fuerzas Armadas en Lima, Perú, 2012: estudio transversal. Medwave. 2016; 16(3): e6435.

32. Rodríguez J, Zapatero G, Martín R. Prevalencia de la depresión en la diabetes mellitus tipo 2. Rev Clin Esp. 2015; 215(3): 156-64.

\begin{tabular}{|l|}
\hline Recibido: 01/04/2019 \\
Aceptado: 02/09/2019 \\
\hline
\end{tabular}

